Modern Physics Letters A, Vol. 11, No. 14 (1996) 1199

(C) World Scientific Publishing Company

ERRATA

\title{
NUMERICAL COMPUTATION OF THE EFFECTIVE POTENTIAL AND RENORMALIZATION
}

\author{
[Mod. Phys. Lett. A, Vol. 11, No. 4 (1996) 281-287]
}

G. Palma and L. Vergara

On p. 283 Eq. (3.1) should read

$$
\begin{aligned}
\hat{U}_{\mathrm{eff}, T}(\phi)= & -\frac{1}{2} \nu^{2} \phi^{2}+\frac{\lambda}{4 !} \phi^{4}+\frac{T}{2} \sum_{n \neq 0} \int \frac{d^{3} k}{(2 \pi)^{3}} \ln \left[\omega_{n}^{2}+\mathbf{k}^{2}+m^{2}(\phi)\right] \\
& +\frac{T}{2} \int \frac{d^{3} k}{(2 \pi)^{3}} \ln \left[\mathbf{k}^{2}+m_{\mathrm{pl}}^{2}(\phi)\right],
\end{aligned}
$$

where the last two terms on the R.H.S. can be separated into one-loop

$$
\hat{U}_{\mathrm{eff}, T}^{(1)}(\phi)=\frac{T}{2} \sum_{n} \int \frac{d^{3} k}{(2 \pi)^{3}} \ln \left[\omega_{n}^{2}+\mathbf{k}^{2}+m^{2}(\phi)\right] .
$$

